\title{
Metformin Increases Protein Phosphatase 2A Activity in Primary Human Skeletal Muscle Cells Derived from Lean Healthy Participants
}

\author{
Aktham Mestareehi $\mathbb{D}^{1},{ }^{1}$ Xiangmin Zhang ${ }^{D},{ }^{1}$ Berhane Seyoum, ${ }^{2}$ Zaher Msallaty, ${ }^{2}$ \\ Abdullah Mallisho $\left(\mathbb{D}^{2}\right.$ Kyle Jon Burghardt $\left(\mathbb{1},{ }^{3}\right.$ Anjaneyulu Kowluru $\mathbb{D}^{1,4}$ \\ and Zhengping $\mathrm{Yi} \mathbb{( i )}^{1}$ \\ ${ }^{1}$ Department of Pharmaceutical Sciences, College of Pharmacy and Health Sciences, Wayne State University, Detroit, MI 48201, USA \\ ${ }^{2}$ Division of Endocrinology, Wayne State University School of Medicine, Wayne State University, Detroit, MI 48201, USA \\ ${ }^{3}$ Department of Pharmacy Practice, Eugene Applebaum College of Pharmacy/Health Sciences, Wayne State University, Detroit, \\ MI, USA \\ ${ }^{4}$ Program for Translational Research in Diabetes, Biomedical Research Service, John D. Dingell VA Medical Center, Detroit, \\ MI 48201, USA
}

Correspondence should be addressed to Zhengping Yi; zhengping.yi@wayne.edu

Received 25 March 2021; Accepted 16 July 2021; Published 29 July 2021

Academic Editor: Maria Mirabelli

Copyright (c) 2021 Aktham Mestareehi et al. This is an open access article distributed under the Creative Commons Attribution License, which permits unrestricted use, distribution, and reproduction in any medium, provided the original work is properly cited.

\begin{abstract}
Context. Skeletal muscle insulin resistance is one of the primary contributors of type 2 diabetes (T2D). Metformin is the first-line drug for the treatment of T2D. The primary effects of metformin include decreasing glucose production in the liver and decreasing insulin resistance in the skeletal muscle. However, the molecular mechanism of metformin's action in skeletal muscle is not well understood. Protein phosphatase 2A (PP2A), a major serine/threonine protein phosphatase, plays a pivotal role in cellular processes, such as signal transduction, cell proliferation, and apoptosis, and acts through dephosphorylating key signaling molecules such as AKT and AMPK. However, whether PP2A plays a role in metformin-induced insulin sensitivity improvement in human skeletal muscle cells remains to be elucidated. Objective. To investigate if PP2A plays a role in metformin-induced insulin sensitivity improvement in human skeletal muscle cells. Participants. Eight lean insulin-sensitive nondiabetic participants (4 females and 4 males; age: $21.0 \pm 1.0$ years; BMI: $22.0 \pm 0.7 \mathrm{~kg} / \mathrm{m}^{2} ; 2$-hour OGTT: $97.0 \pm 6.0 \mathrm{mg} / \mathrm{dl}$; HbAlc: $5.3 \pm 0.1 \%$; fasting plasma glucose: $87.0 \pm 2.0 \mathrm{mg} / \mathrm{dl} ; M$ value; $11.0 \pm 1.0 \mathrm{mg} / \mathrm{kgBW} / \mathrm{min})$. Design. A hyperinsulinemic-euglycemic clamp was performed to assess insulin sensitivity in human subjects, and skeletal muscle biopsy samples were obtained. Primary human skeletal muscle cells (shown to retain metabolic characteristics of donors) were cultured from these muscle biopsies that included 8 lean insulin-sensitive participants. Cultured cells were expanded, differentiated into myotubes, and treated with $50 \mu \mathrm{M}$ metformin for 24 hours before harvesting. PP2Ac activity was measured by a phosphatase activity assay kit (Millipore) according to the manufacturer's protocol. Results. The results indicated that metformin significantly increased the activity of PP2A in the myotubes for all 8 lean insulin-sensitive nondiabetic participants, and the average fold increase is $1.54 \pm 0.11$ $(P<0.001)$. Conclusions. These results provided the first evidence that metformin can activate PP2A in human skeletal muscle cells derived from lean healthy insulin-sensitive participants and may help to understand metformin's action in skeletal muscle in humans.
\end{abstract}




\section{Introduction}

In the US, $\sim 30$ million individuals have type 2 diabetes (T2D), which is associated with long-term damage of various organs (e.g., eyes, kidneys, nerves, heart, and brain). Insulin resistance in skeletal muscle is one of the main causes for T2D [1]. Metformin (N,N-dimethylbiguanide) is an effective oral biguanide antihyperglycemic drug and the most frequently prescribed as a first-line therapy for T2D [2]. Metformin reduces glucose production by the liver and increases insulin sensitivity (i.e., decreased insulin resistance) in skeletal muscles $[2,3]$. In addition, links between insulin resistance, diabetes, and brain disorders have been reported [4, 5], and metformin has been shown to have antiseizure effects in epilepsy animal models and is proposed as a potential candidate for drug repurposing for epilepsy patients [5-7]. Nonetheless, molecular mechanisms of metformin's action in skeletal muscle are incompletely understood. Protein phosphatase 2A (PP2A) is a ubiquitously expressed serine/threonine phosphatase and plays a pivotal role in cellular processes, such as insulin signal transduction, cell proliferation, and apoptosis, by dephosphorylating key signaling molecules such as AKT, AMPK, p53, and c-Myc [8]. Metformin may activate PP2A $[9,10]$, while others have shown that metformin has no effect on PP2A [11]. Whether metformin affects PP2A activity in primary human skeletal muscle cells remains to be elucidated. The overall objective of this study was to determine whether metformin activates PP2A in primary human skeletal muscle cells.

\section{Materials and Methods}

2.1. Antibodies and Reagents. The PP2A activity assay kit was purchased from EMD Millipore (cat. 17313), which has been successfully used in various studies [12-14]. The PP2A activity assay kit contains protein $\mathrm{A}$ agarose, anti-PP2A, C subunit, clone 1D6 (catalog \# 05-421), normal mouse IgG (catalog \# 12-371), protein A agarose (catalog \# 16-125D), threonine phosphopeptide (K-R-pT-I-R-R) (catalog \# 12-219), pNPP Ser/Thr assay buffer (catalog \# 20-179), Malachite green additive (solution A \& B) (catalog \# 20-105 and catalog \# 20-104), phosphate standard (solution C) (catalog \# 20-103), and 96 -well microtiter plate ( $1 / 2$ volume flat bottom plate). Metformin hydrochloride (Tocris Bioscience, Bristol, UK) was prediluted in purified water as $\times 10$ stock and preserved under $-80^{\circ} \mathrm{C}$ before use. Bradford reagent was purchased from Sigma.

2.2. Experimental Design. The overall experimental design is presented in Supplementary Figure 1. Briefly, extensive subject recruitment was followed by comprehensive screening tests (e.g., vitals, 2-hour oral glucose tolerance test ( $2 \mathrm{~h}$ OGTT), and blood chemistry). Eligible subjects underwent in-patient clinical tests: a skeletal muscle biopsy followed by a 2-hour hyperinsulinemic-euglycemic clamp to assess insulin sensitivity. Biopsies were immediately blotted free of blood and cleaned of connective tissue $(\sim 30 \mathrm{sec})$, submerged in ice-cold DPBS for primary skeletal muscle cell culturing as described in references [15-18]. The primary cells were differentiated into myotubes and treated with/out metformin. The cells are lysed, and protein concentration was measured. Two hundred $\mu \mathrm{g}$ lysate proteins from each sample were utilized for PP2A activity assay. NIgG immunoprecipitation was used to determine the background phosphate activity, and the supernatant from the NIgG immunoprecipitation was subjected to PP2Ac immunoprecipitation. Both NIgG and PP2Ac immunoprecipitates were incubated with the phosphatase substrate (i.e., threonine phosphopeptide (K-R-pT-I-R-R)), and the resulting supernatants were mixed with Malachite green phosphate detection solution, followed by the measurement of the absorbance at $650 \mathrm{~nm}$. The amount of free phosphate released (reflecting phosphatase activity) was calculated from a standard curve. The resulting PP2A activity data were integrated with clinical data.

2.3. The Clinical Studies and Subjects. This protocol was approved by the Institutional Review Board of Wayne State University. The clinical studies started with participant recruitment, followed by comprehensive screening tests (Visit 1) and involved hyperinsulinemic-euglycemic clamp and muscle biopsies (Visit 2). All participants were instructed to stop any form of exercise for at least 2 days before each visit, and none of them had any significant medical problems. On Visit 1 day, the subject arrived at the clinical research service center in the morning after a 10-hour overnight fast. Written consent was obtained before their participation, and the purpose and potential risks of the study were explained to all participants. Measurement of vitals, urine analysis, 2-hour oral glucose tolerance test (OGTT), pregnancy test if female participant, and 12-lead electrocardiogram (ECG) were performed at site. HbAlc and blood chemistry were measured by the Detroit medical center (DMC). Eligible participants (a total of 8 volunteers) were scheduled for Visit 2.

2.4. Muscle Biopsy and Hyperinsulinemic-Euglycemic Clamp. The hyperinsulinemic-euglycemic clamp is considered to be the gold standard for measuring insulin sensitivity in vivo as previously described [18-20]. The study began at approximately 8 am (time $-60 \mathrm{~min}$ ) after an overnight fast. A catheter was placed in an antecubital vein and maintained throughout the study for infusions of insulin and glucose. A second catheter was placed in a vein in the contralateral arm for the sampling of arterialized venous blood. Blood glucose was measured and reported every 15 minutes before insulin infusion started and every 5 minutes after insulin infusion started. Around 8:30 am (time $-30 \mathrm{~min}$ ), a licensed physician performed the muscle biopsies from the vastus lateralis using the modified Bergstrom technique under local anesthesia (lidocaine) [21]. The collected biopsies were immediately blotted free of blood and cleaned of connective tissue and fat $(\sim 30 \mathrm{sec})$ and submerged in ice-cold media and transported to the laboratory for primary skeletal muscle cell culturing as described previously [15-17]. At $\sim 9$ am (time $0 \mathrm{~min}$ ), a primed, continuous infusion of human regular insulin (Humulin R; Eli Lilly, Indianapolis, IN) was initiated and continued for 120 minutes to quantify the effects of insulin on glucose disposal. Insulin infusion was started at 
$160 \mathrm{mU} /\left(\mathrm{m}^{2} \cdot \mathrm{min}\right)$ for 5 minutes, followed by $120 \mathrm{mU} /\left(\mathrm{m}^{2} \cdot\right.$ min) for 5 minutes, and then maintained at constant $80 \mathrm{mU} /\left(\mathrm{m}^{2} \cdot \mathrm{min}\right)$. This ensured that steady-state levels were more rapidly achieved, minimizing the burden on the participants. Throughout the insulin infusion, an infusion of $20 \%$ glucose was adjusted to maintain euglycemia (targeted at $90 \mathrm{mg} / \mathrm{dl}$ ). Insulin-stimulated glucose disposal rate ( $M$ value) was calculated as the average glucose infusion rate value during the final $30 \mathrm{~min}$ of insulin infusion.

2.5. Primary Cell Cultures and Treatments. The biopsy was washed $3 \mathrm{x}$ in ice-cold phosphate buffer saline (PBS) and minced into small pieces with $0.05 \%$ Trypsin-EDTA added. The minced tissues were centrifuged and filtered through a nylon mesh. The resulting human skeletal muscle cells were cultured in growth medium (Dulbecco modified Eagle's medium) supplemented with $10 \%$ FBS, $1 \%$ PSG, $10 \mathrm{ng} / \mathrm{ml}$ EGF, $0.4 \mu \mathrm{g} / \mathrm{ml}$ dexamethasone, $50 \mu \mathrm{g} / \mathrm{ml}$ fetuin, $1 \%$ sodium pyruvate, and $1 \%$ NEAA and maintained in a humidified atmosphere at $37^{\circ} \mathrm{C}$ and $5 \% \mathrm{CO}_{2}$. The human skeletal muscle cells expanded, and the culture medium was completely changed every other day. The myoblasts were differentiated into myotubes and treated with or without $50 \mu \mathrm{M}$ metformin for 24 hours as described in the Supplementary Materials.

2.6. PP2A Activity Assay. The PP2A activity was measured according to the manufacturer's protocol. The cells were harvested and homogenized in $1 \mathrm{ml}$ of lysis activity buffer included $(20 \mathrm{mM}$ imidazole- $\mathrm{HCl}, 2 \mathrm{mM}$ EDTA, $2 \mathrm{mM}$ EGTA, pH 7.0, with $10 \mu \mathrm{g} / \mathrm{ml}$ each of aprotinin, leupeptin, $1 \mathrm{mM}$ benzamidine, and $1 \mathrm{mM}$ PMSF). The cells were collected in Eppendorf tubes, homogenized for 10 minutes at $4^{\circ} \mathrm{C}$, and centrifuged at $11000 \times \mathrm{g}$ for 15 minutes at $4^{\circ} \mathrm{C}$. The protein concentration was measured using Bradford protein assay method. $200 \mu \mathrm{g}$ of total protein of each sample was transferred to new Eppendorf tubes, including $30 \mu \mathrm{l}$ of protein $\mathrm{A}$ agarose and $4 \mu \mathrm{l}$ of normal mouse IgG, and the volumes were topped off to $1000 \mu \mathrm{l}$ with pNPP assay buffer. The NIgG immunoprecipitates were incubated for one and half hour at $4^{\circ} \mathrm{C}$ with constant rocking and followed by centrifugation at $3000 \times \mathrm{g}$ for 3 minutes at $4^{\circ} \mathrm{C}$. The supernatants were transferred to new Eppendorf tubes, including $30 \mu \mathrm{l}$ of protein A agarose and $4 \mu \mathrm{l}$ of anti-PP2Ac antibody, and the volumes were topped off to $1000 \mu \mathrm{l}$ with pNPP assay buffer. All tubes were incubated at constant rotated for 2 hours at $4^{\circ} \mathrm{C}$ and centrifuged at $3000 \times \mathrm{g}$ for 3 minutes at $4^{\circ} \mathrm{C}$. The immunoprecipitated PP2Ac and NIgG beads were washed 3 times with $700 \mu \mathrm{l}$ Tris-buffered saline (TBS) $(3000 \times \mathrm{g}$ for 3 minutes at $4^{\circ} \mathrm{C}$ ), once with $500 \mu \mathrm{l} \mathrm{pNPP}$ Ser/Thr assay buffer, followed by incubation with $60 \mu \mathrm{l}$ of $750 \mu \mathrm{M}$ of threonine phosphopeptide (K-R-pT-I-R-R) and $20 \mu \mathrm{l}$ of pNPP Ser/Thr assay buffer at $30^{\circ} \mathrm{C}$ for 10 minutes in a shaking incubator. The beads were centrifuged $3000 \times \mathrm{g}$ for 3 minutes at $4^{\circ} \mathrm{C}$, and $25 \mu \mathrm{l}$ of the supernatants was mixed with $100 \mu \mathrm{l}$ of Malachite green phosphate detection solution into each well to a 96-well plate microtiter plate to terminate the reaction, and the color was allowed to develop for 15 minutes at room temperature. The absorbance of the samples was read using a microplate reader at $650 \mathrm{~nm}$. The amount of free phosphate released was calculated from a standard curve (according to the manufacturer's protocol) and normalized to that of the controls.

2.7. Statistical Analysis. All data were represented as the mean \pm standard error of the mean (SEM) from eight independent experiments. The significances between the control and metformin-treated groups were analyzed by independent Student's $t$-test.

\section{Results}

We enrolled 8 lean healthy human subjects. A hyperinsulinemiceuglycemic clamp was performed to assess insulin sensitivity, and skeletal muscle biopsy samples were obtained. As can be seen from Supplementary Table 1, these participants are healthy, without family history of $\mathrm{T} 2 \mathrm{D}$, and they are nondiabetic, non-prediabetic with normal $\mathrm{HbAlc}$, normal fasting plasma glucose, normal glucose tolerant, and high insulin sensitivity.

Primary human skeletal muscle cells were cultured from these muscle biopsies, expanded, differentiated into myotubes, and treated with/without $50 \mu \mathrm{M}$ metformin for 24 hours. Myotubes were homogenized in phosphate free buffer, followed by PP2A activity assay. A standard curve was generated using the standard phosphopeptide (Figure 1(a) with $R^{2}$ at 0.996 ). The slope and intercept were used to calculate the amount of phosphate released by the PP2Ac immunoprecipitates incubated with the standard phosphopeptide. Negligible PP2Ac activity in the NIgG immunoprecipitation samples was detected, indicating our assays were not contaminated with exogenous phosphate or nonspecific immunoprecipitates. PP2A activity measurement indicated that metformin increased the activity of PP2A in the myotubes derived from all eight lean nondiabetic participants as presented in Figures 1(b) and 1(c) (fold increase metformin/basal: $1.54 \pm 0.11, P<0.001)$. These results provided the first evidence that metformin promotes activation of PP2A in human skeletal muscle cells derived from lean, insulin-sensitive, nondiabetic participants.

Correlation analysis of PP2A activity (either with or without metformin treatment) with participants' clinical characteristics indicated that PP2A activity has significant correlation with none of the clinical characteristics for the 8 lean healthy participants except for the heart rate (Supplementary Table 2). One possible explanation is that the participants in this study were homogeneous and all of them are lean, young, and healthy with high insulin sensitivity. Whether PP2A activity has significant correlations with clinical characteristics in obese insulin-resistant participants or T2D patients warrants further investigation.

\section{Discussion}

The molecular mechanisms of metformin are not fully understood. Metformin inhibits complex I in the mitochondrial electron transport chain in liver and muscle cells as well as skeletal muscle [22]. Another potential molecular 


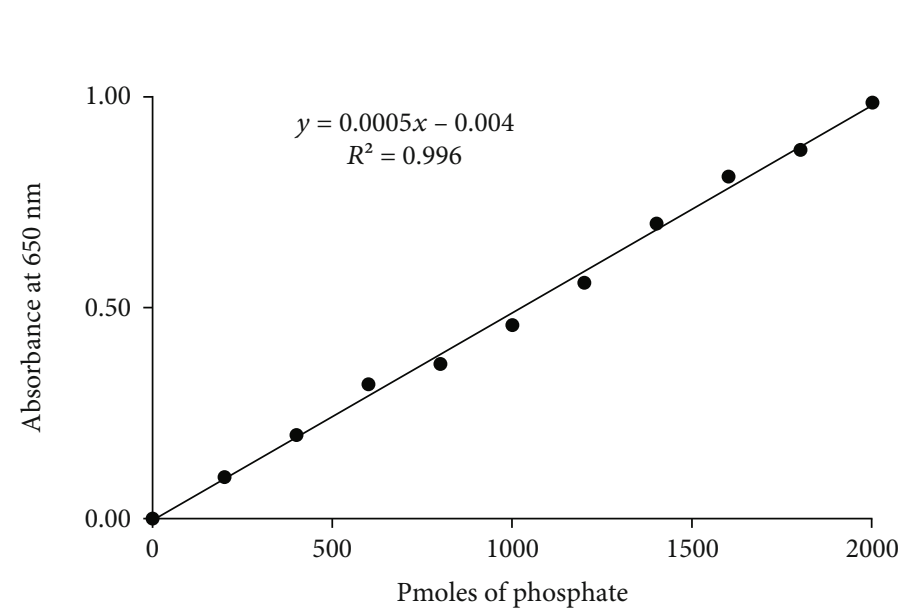

(a)

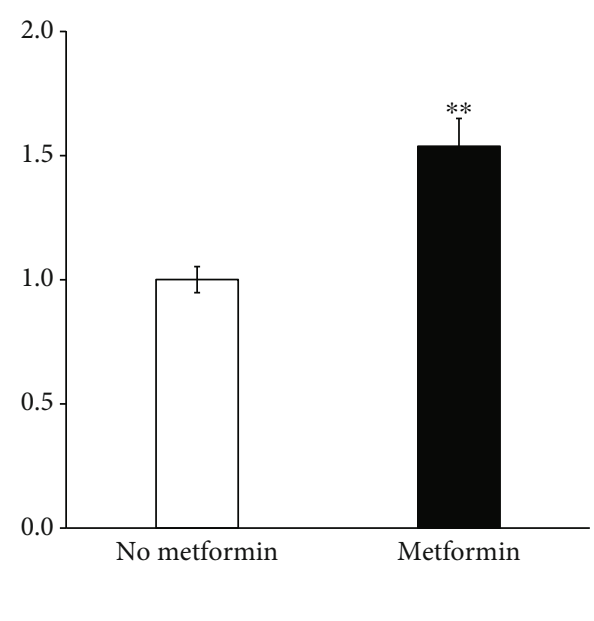

(b)

\begin{tabular}{|c|c|c|c|c|c|c|c|c|c|c|c|}
\hline Participant \# & 1 & 2 & 3 & 4 & 5 & 6 & 7 & 8 & Mean & SEM & $P$ value \\
\hline \multicolumn{12}{|c|}{ Amount of phosphate released by the PP2Ac immunoprecipitates (pmole) $)^{\#}$} \\
\hline No metformin & 1089 & 1221 & 1255 & 1333 & 1638 & 1256 & 1217 & 1009 & 1252 & 66 & \\
\hline Metformin & 1716 & 1866 & 1872 & 1767 & 1855 & 1816 & 2881 & 1620 & 1924 & 140 & 0.0007 \\
\hline \multicolumn{12}{|c|}{ Normalized PP2A activity } \\
\hline No metformin & 0.87 & 0.98 & 1.00 & 1.06 & 1.31 & 1.00 & 0.97 & 0.81 & 1.00 & 0.05 & \\
\hline Metformin & 1.37 & 1.49 & 1.50 & 1.41 & 1.48 & 1.45 & 2.30 & 1.29 & 1.54 & 0.11 & 0.0007 \\
\hline
\end{tabular}

FIGURE 1: Effect of metformin on PP2A activity in primary human muscle cells derived from 8 lean insulin-sensitive nondiabetic participants $(n=8)$. (a) Measurement of phosphate concentration of the standard solutions (according to the manufacturer's protocol). (b) Summative PP2A activity. Data are given as fold changes (means \pm SEM). The mean of the PP2A activity for the 8 samples without metformin treatment was set to 1.00 , and the fold changes were relative to basal (i.e., no metformin treatment). (c) Individual PP2A activity. The significance between the basal and metformin-treated groups was analyzed by independent Student's $t$-test. ${ }^{* *} P<0.001 .{ }^{*}$ Amount of phosphate released reflects the PP2A activity, and the mean of the PP2A activity for the 8 samples without metformin treatment was set to 1.00 .

mechanism is the activation of AMPK in skeletal muscle after metformin treatment [3].

Multiple studies have reported the effect of metformin on PP2A activity. Deepa et al. reported that metformin has no effect on PP2A activity in C2C12 myotube (a mouse cell line) [11]. On the other hand, compatible with our results, several studies have demonstrated that metformin induced PP2A activity in several cell systems. For example, in lung cancer cells (A549 and H1651), metformin-activated PP2A reduced tumor formation in vivo and decreased tumor cell growth and invasion capacity in vitro as well as serine phosphorylation level of Bax (Ser184), Myc (Ser62), and Akt (Ser473) [23]. Sacco et al. reported that metformin treatment induced the protein expression of PP2A catalytic and regulatory subunit in breast cancer cells in vitro [24]. Notably, Auger et al. have reported that metformin activated PP2A in human subcutaneous white adipose tissue (scWAT), resulting in dephosphorylation of acetyl-CoA carboxylase (Ser79) and hormone-sensitive lipase (Ser660), and metformin also lowered lipolysis in beige fat [25]. Kawashima et al. demonstrated that metformin treatment activated PP2A in myeloproliferative neoplasm (MPN) cells to suppress the oncogenic kinase JAK2V617F by increasing reactive oxygen species levels leading to the inhibition of SHP-2, a positive regulator of JAK2V617F; furthermore, the results indicated that metformin suppressed phosphorylation of the PP2Ac at Tyr307 in a dose-dependent manner. These results revealed that metformin activated PP2A independent of AMPK activation [26]. PP2A is one of the major tau phosphatases, and metformin increased PP2A activity and reduced tau phosphorylation at PP2A-dependent epitopes in vitro and in vivo from primary cortical neuron cells [10]. Metformin treatments induced PRKN gene transcription, mitochondria integrity, mitophagy, and cell viability and decreased activation of nuclear factor kappa B (NF- $\kappa$ B) but not of p53 or ATF4 in human renal epithelial cells [27]. Zhang et al. reported that metformin decreased NLRP3 protein expression and NLRP3 inflammasome activation in ox-LDL-stimulated macrophages through AMPK and PP2A. PP2A catalytic activity was required for NF- $\kappa$ B inhibition and tristetraprolin activation induced by metformin in ox-LDL-stimulated macrophages [9].

Despite the evidence, albeit indirect, on potential regulation of protein phosphorylation-dephosphorylation of key cellular proteins and functions by metformin, putative mechanisms underlying metformin-mediated activation of PP2A remain unclear at this time. $\mathrm{PP} 2 \mathrm{~A}$ is regulated by the $\mathrm{mTOR}$ kinase, and both enzymes affect the phosphorylation status of the ribosomal protein S6 and p70S6K. PP2A has been shown to dephosphorylate the mTOR substrate S6K1 and involved 
in mTOR-mediated phosphorylation of insulin receptor substrate 1 through modulation of PP2A activity by mTOR [28]. Along these lines, Kickstein and coworkers have reported that metformin directly affected PP2A independently of AMPK/TOR signaling [10]. Based on the data accrued in these studies, the authors have proposed that metformin interferes with association of PP2Ac with MID1- $\alpha 4$ complex leading to regulation of PP2A activity. On the other hand, activation or phosphorylation of AMPK by metformin leads to the inhibition of mTOR singling and subsequent dissociation of PP2A and $\alpha 4$ and resulting in PP2A activation [29]. It has also been proposed that metformin could dissociate the binding of PP2A and MID1 resulting in decreased PP2A degradation and enhanced phosphatase activity $[10,29]$. Additionally, it is likely that metformin could promote interaction between the structural (A subunit), regulatory (B subunit), and catalytic $\mathrm{C}$ subunits leading to functional activation of the enzyme. Such interactions may be influenced by posttranslational modification of individual subunits, including the methylation of the catalytic subunit at Leucine-309 residue [8], which has been shown to promote PP2A activation. Future studies will address these aspects to provide mechanical insights into metformin-induced PP2A activation that we report in the current study.

\section{Conclusions}

In the present study, we demonstrated that metformin significantly increased PP2A activity in the myotubes derived from all eight lean nondiabetic participants with high insulin sensitivity. These results provided the first evidence to suggest that metformin promotes activation of $\mathrm{PP} 2 \mathrm{~A}$ in human skeletal muscle cells and might aid potential new targets for novel mechanistic studies on skeletal muscle insulin resistance in humans.

\section{Abbreviations}

AMPK: AMP-activated protein kinase

AKT: $\quad$ Serine/threonine protein kinase B (PKB)

mTOR: Mammalian target of rapamycin

PP2A: Serine/threonine protein phosphatase 2A

NIgG: Normal mouse IgG

PP2Ac: PP2A catalytic subunit

MPN: Myeloproliferative neoplasm

NF- $\kappa$ B: Nuclear factor kappa B

EDTA: Ethylenediaminetetraacetic acid

FBS: Fetal bovine serum

EGTA: Ethylene glycol bis (2-aminoethyl ether)-N,N, ${ }^{\prime} \mathrm{N}^{\prime}$ tetraacetic acid.

\section{Data Availability}

The Supplementary Tables data used to support the findings of this study are included within the supplementary information file.

\section{Disclosure}

The contents of the article were presented in a poster presentation: Integrated Physiology/Obesity at the $80^{\text {th }}$ American Diabetes Association meeting.

\section{Conflicts of Interest}

The authors declare no conflict of interest and have nothing to disclose.

\section{Acknowledgments}

This work was in part supported by NIH/NIDDK, R01 DK107666 (ZY) and R01 DK081750 (ZY), American Diabetes Association Translational Science award \#1-13-TS-27 and Clinical/Translational award \#7-09-CT-56 (ZY), Detroit Cardiovascular Training Program T32HL120822 (AM/AK/ZY), a Merit Review Award (I01BX004663-01) and the Senior Research Career Scientist Award (IK6BX005383) from the U.S. Department of Veterans Affairs (AK), Wayne State University Diabetes and Obesity Team Science (DOTS, ZY), and DOTS scholars (AM/ZY).

\section{Supplementary Materials}

Supplementary information includes Supplementary Figure 1, Supplementary Table 1, and Supplementary Table 2 as well as supplementary method. Supplementary 1 Supplementary Table 1: clinical characteristics of lean nondiabetic participants in the study. All measurements were done after an overnight fast. Supplementary Table 2: correlation of PP2A activity (either with or without metformin treatment) with participants' clinical characteristics. Supplementary 2 Supplementary Figure 1: schematic diagram of clinical and biological studies. (Supplementary Materials)

\section{References}

[1] J. R. Zierath, A. Krook, and H. Wallberg-Henriksson, "Insulin action and insulin resistance in human skeletal muscle," Diabetologia, vol. 43, no. 7, pp. 821-835, 2000.

[2] R. W. Hunter, C. C. Hughey, L. Lantier et al., "Metformin reduces liver glucose production by inhibition of fructose-16-bisphosphatase," Nature Medicine, vol. 24, no. 9, pp. 13951406, 2018.

[3] N. Musi, M. F. Hirshman, J. Nygren et al., "Metformin increases AMP-activated protein kinase activity in skeletal muscle of subjects with type 2 diabetes," Diabetes, vol. 51, no. 7, pp. 2074-2081, 2002.

[4] N. A. Shlobin and J. W. Sander, "Drivers for the comorbidity of type 2 diabetes mellitus and epilepsy: a scoping review," Epilepsy \& Behavior, vol. 106, p. 107043, 2020.

[5] V. Nesci, E. Russo, B. Arcidiacono et al., "Metabolic alterations predispose to seizure development in high-fat diet-treated mice: the role of metformin," Molecular Neurobiology, vol. 57, no. 11, pp. 4778-4789, 2020.

[6] E. M. Yimer, A. Surur, D. Z. Wondafrash, and A. K. Gebre, "The effect of metformin in experimentally induced animal models of epileptic seizure," Behavioural Neurology, vol. 2019, Article ID 6234758, 13 pages, 2019. 
[7] L. Brueggeman, M. L. Sturgeon, R. M. Martin et al., "Drug repositioning in epilepsy reveals novel antiseizure candidates," Annals of Clinical Translational Neurology, vol. 6, no. 2, pp. 295-309, 2019.

[8] A. Kowluru and A. Matti, "Hyperactivation of protein phosphatase $2 \mathrm{~A}$ in models of glucolipotoxicity and diabetes: potential mechanisms and functional consequences," Biochemical Pharmacology, vol. 84, no. 5, pp. 591-597, 2012.

[9] L. Zhang, L. Lu, X. Zhong et al., "Metformin reduced NLRP3 inflammasome activity in Ox-LDL stimulated macrophages through adenosine monophosphate activated protein kinase and protein phosphatase 2A," European Journal of Pharmacology, vol. 852, pp. 99-106, 2019.

[10] E. Kickstein, S. Krauss, P. Thornhill et al., "Biguanide metformin acts on tau phosphorylation via $\mathrm{mTOR} /$ protein phosphatase 2A (PP2A) signaling," Proceedings of the National Academy of Sciences of the United States of America, vol. 107, no. 50 , pp. $21830-21835,2010$.

[11] S. S. Deepa, L. Zhou, J. Ryu et al., "APPL1 mediates adiponectin-induced LKB1 cytosolic localization through the PP2A-PKCzeta signaling pathway," Molecular Endocrinology, vol. 25, no. 10, pp. 1773-1785, 2011.

[12] A. M. Wallace, A. Hardigan, P. Geraghty et al., "Protein phosphatase $2 \mathrm{~A}$ regulates innate immune and proteolytic responses to cigarette smoke exposure in the lung," Toxicological sciences : an official journal of the Society of Toxicology, vol. 126, no. 2, pp. 589-599, 2012.

[13] G. Cheng, H. Kasiganesan, C. F. Baicu, J. G. Wallenborn, D. Kuppuswamy, and G. Cooper, "Cytoskeletal role in protection of the failing heart by $\beta$-adrenergic blockade," American Journal of Physiology Heart and Circulatory Physiology, vol. 302, no. 3, pp. H675-H687, 2012.

[14] A. Rahman, B. J. Brew, and G. J. Guillemin, "Lead dysregulates serine/threonine protein phosphatases in human neurons," Neurochemical Research, vol. 36, no. 2, pp. 195-204, 2011.

[15] D. B. Thompson, R. Pratley, and V. Ossowski, "Human primary myoblast cell cultures from non-diabetic insulin resistant subjects retain defects in insulin action," The Journal of Clinical Investigation, vol. 98, no. 10, pp. 2346-2350, 1996.

[16] R. R. Henry, L. Abrams, S. Nikoulina, and T. P. Ciaraldi, "Insulin action and glucose metabolism in nondiabetic control and NIDDM subjects. Comparison using human skeletal muscle cell cultures," Diabetes, vol. 44, no. 8, pp. 936-946, 1995.

[17] V. Bourlier, C. Saint-Laurent, K. Louche et al., "Enhanced glucose metabolism is preserved in cultured primary myotubes from obese donors in response to exercise training," The Journal of Clinical Endocrinology and Metabolism, vol. 98, no. 9, pp. 3739-3747, 2013.

[18] Y. Qi, X. Zhang, B. Seyoum et al., "Kinome profiling reveals abnormal activity of kinases in skeletal muscle from adults with obesity and insulin resistance," The Journal of Clinical Endocrinology and Metabolism, vol. 105, no. 3, pp. 644-659, 2020.

[19] R. A. DeFronzo and D. Tripathy, "Skeletal muscle insulin resistance is the primary defect in type 2 diabetes," Diabetes Care, vol. 32, suppl_2, pp. S157-S163, 2009.

[20] M. Caruso, D. Ma, Z. Msallaty et al., "Increased interaction with insulin receptor substrate 1 , a novel abnormality in insulin resistance and type 2 diabetes," Diabetes, vol. 63, no. 6, pp. 1933-1947, 2014.
[21] R. A. Shanely, K. A. Zwetsloot, N. T. Triplett, M. P. Meaney, G. E. Farris, and D. C. Nieman, "Human skeletal muscle biopsy procedures using the modified Bergstrom technique," Journal of visualized experiments : JoVE, vol. 91, article 51812, 2014.

[22] M. R. Owen, E. Doran, and A. P. Halestrap, "Evidence that metformin exerts its anti-diabetic effects through inhibition of complex 1 of the mitochondrial respiratory chain," The Biochemical Journal, vol. 348, no. 3, pp. 607-614, 2000.

[23] X. Zhou, S. Liu, X. Lin et al., "Metformin inhibit lung cancer cell growth and invasion in vitro as well as tumor formation in vivo partially by activating PP2A," Medical Science Monitor : International Medical Journal of Experimental and Clinical Research, vol. 25, pp. 836-846, 2019.

[24] F. Sacco, A. Silvestri, D. Posca et al., "Deep proteomics of breast cancer cells reveals that metformin rewires signaling networks away from a pro-growth state," Cell Systems, vol. 2, no. 3, pp. 159-171, 2016.

[25] C. Auger, C. M. Knuth, A. Abdullahi, O. Samadi, A. Parousis, and M. G. Jeschke, "Metformin prevents the pathological browning of subcutaneous white adipose tissue," Molecular Metabolism, vol. 29, pp. 12-23, 2019.

[26] I. Kawashima and K. Kirito, "Metformin inhibits JAK2V617F activity in MPN cells by activating AMPK and PP2A complexes containing the B56 $\alpha$ subunit," The Journal of Clinical Investigation, vol. 44, no. 12, pp. 1156-1165.e4, 2016.

[27] Y. Zhao and M. Sun, "Metformin rescues Parkin protein expression and mitophagy in high glucose- challenged human renal epithelial cells by inhibiting NF- $\kappa$ B via PP2A activation," Life Sciences, vol. 246, p. 117382, 2020.

[28] D. Hartley and G. M. Cooper, "Role of mTOR in the degradation of IRS-1: regulation of PP2A activity," Journal of Cellular Biochemistry, vol. 85, no. 2, pp. 304-314, 2002.

[29] R. J. Dowling, M. Zakikhani, I. G. Fantus, M. Pollak, and N. Sonenberg, "Metformin inhibits mammalian target of rapamycin-dependent translation initiation in breast cancer cells," Cancer Research, vol. 67, no. 22, pp. 10804-10812, 2007. 